\title{
A portfolio approach for the selection and the timing of urban planning projects
}

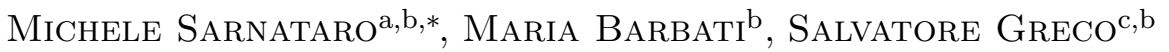 \\ ${ }^{a}$ Department of Civil, Architectural and Environmental Engineering, University of \\ Naples Federico II, Naples, Italy \\ ${ }^{b}$ Portsmouth Business School, Operations and Systems Management Group, Centre of \\ Operations Research and Logistics (CORL), \\ University of Portsmouth, Portsmouth, United Kingdom \\ ${ }^{c}$ Department of Economics and Business, University of Catania, Italy
}

\begin{abstract}
This paper presents a model to support organizations dealing with urban planning decisions. In particular, we deal with the selection and the timing of several projects that can be realised within an urban development project with the aim of optimizing conflicting objectives. The available resources should be allocated in an acceptable way and current and future requirements must be met. In addition, projects can be characterized by qualitative performances and different scenarios can be considered.

The model can be handled with different methods. Given its participatory features, it seems that an interactive multiobjective optimization methodology is the most appropriate approach, since the Decision Maker can express his opinion throughout the resolution process.

We present how the model can work through the description of an application based on the execution of the Master Plan of the University of Portsmouth, one of the most fast progressing University in UK. We show

*Corresponding author at: Richmond Building, Portland Street, Portsmouth, PO13DE, UK

Email address: michele.sarnat@gmail.com (Michele SARnATARo)
\end{abstract}


the versatility of the model and how it can successfully handle such complex decisions.

Keywords: Multiple Objectives Optimization, Portfolio Decision Analysis, Urban Planning, Scheduling

\section{Introduction}

Urban planning projects are key strategic decisions that require a considerable amount of time and money to ensure the success of the initiatives. In the current economic context, the scarcity of financial resources, especially from public authorities, together with the duty to meet challenging strategic objectives, implies an even more considerable effort to decide which projects are the best to select and in which period they should be planned [1]. Usually, urban development processes have a big impact on several areas of the city, even shaping its social, urban and economic structure [2]. Therefore, several stakeholders are involved, including not only the organizations implementing the projects, but also the citizens and, more in general, the public opinion [3]. Because of these different and multiple interests, it is evident that not only monetary aspects can be valued [4] but a variety of objectives needs to be optimized. Moreover, such complicated perspectives are even more challenging for the presence of high uncertainty scenarios and imprecise assessments that may change over time [5]. Furthermore, in urban planning, the evaluations of the various projects are subject to uncertainty and inaccurate determination, and more detailed information will be released only in successive periods. As consequence, not only the selection of the projects needs to be made at the beginning of a planning period, but it is also necessary to define the timing of those projects in order to distribute the available budget among the projects in a timely manner. Hence, 
in urban planning it is essential to consider the following elements:

- An urban plan can be defined as a selection of a portfolio of urban projects according to the available financial resources and strategic needs to meet.

- The evaluation of the projects changes over time and depends on the time in which the project will be implemented; thus, the decision must concern also the temporal distribution, i.e. the scheduling, of such projects.

- Several objectives need to be optimized. The evaluation of each project for each objective may be not exactly defined, and qualitative evaluations of the contribution of each project to each objective may be necessary, especially in the initial planning stage.

- There is uncertainty in regards to the financial resources available and for the strategic needs to meet. Consequently, there must be the possibility of dealing with unforeseen circumstances and simulate different scenarios.

- Several stakeholders, including people living in and around the urban space where projects are going to be implemented, are involved. For this reason, a participatory approach should be implemented.

In this paper we present a multiobjective model for selecting and timing portfolios of urban projects permitting consideration of uncertainty, qualitative evaluations and temporal distribution of the benefits of the projects. We propose to find good compromise portfolios thanks to an interactive 
method that allows the active participation of the stakeholders in the construction of the decision to take. The stakeholders can develop the portfolio strategy in a participative form even revising their own preferences during a structured decision process oriented to the progressive construction of the solution. Our approach is characterized by the following innovative points:

- We define urban planning problems as a portfolio selection and timing problem. This allows us to focus the attention only on the most urgent urban projects and to schedule them at the most appropriate time.

- We can fully represent different points of view, that it is essential when dealing with such complex problems. Indeed, our problem has a multiobjective formulation that can consider a variety of objectives.

- We are able to handle qualitative information thanks to the introduction of qualitative thresholds for each criterion introduced. This implies that even vague evaluations of projects in the early planning stage can be considered in the selection and timing process.

- We can deal with uncertain conditions, thanks to the possibility of taking into account the stochastic nature of the variables related to the ever-changing urban conditions.

- We can take into account a variety of budget and planning constraints. Several salient aspects can be easily formulated by analysts representing perception of the problems of the different stakeholders. Such constraints can also be modified when the conditions in which the planning is taking place change.

- We introduce a structured participatory method that allows the stakeholders to construct the solution within an interactive and transparent 
procedure. The preferences of the stakeholders are taken into account throughout the whole process and a sensible compromise can be found among the different interests.

To show the benefits of our model and method, we illustrate our methodology presenting a possible application to the selection of the Master Plan projects of the University of Portsmouth, a fast growing University in UK, located in the city centre of the second highest density city in UK.

The paper is organized as follows. In the next Section we briefly review the literature concerning multiobjective optimization portfolio problems for urban planning problems. In Section 3 we introduce the model, while in Section 4 we illustrate the interactive multiobjective optimization method used to tackle the model. In Section 5 we introduce the case study and we show how the model and the method can be adapted to this case. Finally, Section 6 concludes the paper.

\section{Multiobjectives portfolio selection problems and urban plan- ning}

Multiobjective optimization problems are formulated and solved with the aim to support stakeholders in determining a solution that best fits their preferences when several and conflicting objectives need to be optimized [6]. The portfolio decision analysis [7] deals with the selection of a set of projects according to one or more objectives to optimize and with some constraints to take into account. When more objectives are optimized, such as environmental objectives together with economic objectives, multiobjective portfolio problems can be formulated [8]. Urban planning can be described as the process of defining urban development through the im- 
plementation of urban projects, such as physical plans, that aim to make the urban area a better place from a social, economic and environmental points of view [9]. Therefore, urban planning can represent a multiobjective portfolio problem in the sense of selecting a set of urban projects, subject to a set of urban and budgetary constraints and where several objectives must be taken into account [10]. Therefore, a multiobjective portfolio selection model can be used to describe the selection of urban projects according not only to economic factors but also to several other objectives such as preserving the quality of the environment and guaranteeing the sustainability of the projects [11]. Moreover, because the selection process considers the whole set of projects to be defined altogether, the effects that the whole portfolio have on the different objectives [7] make the entire urban plan process more comprehensive.

Multiobjective approaches have been used for evaluating and selecting urban projects in many different real world applications (e.g. [12] or [13]). Those approaches support decisions concerning different interventions in the urban ambit varying from the design of urban spaces (i.e. positioning distribution centers [14] or selecting Parking Lot Site [15]), to the definition of management strategies concerning the possible uses of the different areas of the cities [16]. Indeed, several types of methodologies can be adopted. For example, evolutionary algorithms such as NSGA-II [17] have been applied to define how to allocate different land uses [18], or to define where to locate charging points for the electric vehicles [19]. Another example is the adoption of a multiobjective Tabu Search to define the layout of emergency hospitals [20]. Let us highlight that many of those approaches focus on the selection of only one most preferred project or on the definition of a ranking of a set of projects [21]. 
Nonetheless, a few examples that consider the whole portfolio can be retrieved in the literature. [22] combines a multicriteria method with a genetic algorithm to build a framework for the selection of sustainable urban development projects, while [23] provides a general framework for the selection of projects in environmental management and in particular for the selection of urban water services.

An interesting area of applications is related to the interventions to preserve the cultural heritage and in particular to the regeneration of areas or buildings. In this context, [24] proposed to generate a plan that chooses a portfolio of interventions concerning cultural heritage projects to be made altogether. The same authors adopted a similar approach for a different case study [2]. Recently, in the work proposed by [8], a methodology is developed for the selection or cultural heritage projects by the continuous interaction with the different stakeholders.

Let us highlight that in all the above cases, different methods have been used to find the most preferred solutions, but, to the best of our knowledge, interactive methods are rarely used [25]. Moreover, even if an interaction with the Decision Maker (DM) is included, it is often implemented as a judgement of the DM on the set of the so called Pareto optimal portfolios, which implies a huge cognitive burden for him. Furthermore, the interaction is not conducted through properly structured methods that include preference information of the DM in the resolution process (i.e. asking the DM to judge portfolios by means of only the interactive visual analysis [26]).

Let us also note that, those multiobjective portfolio optimization approaches for urban planning projects, give very small attention to the timing of the projects. In the literature, there are several approaches that deal with the project portfolio selection and timing in different contexts (see e.g., [27] 
and in a subsequent evolution [28]). Several different aspects have been analyzed as the interdependency of the projects [29], the modeling of synergies and incompatibilities among projects and uncertainty in the parameters of the problem [30], or the possibility of interrupting projects even once they have been planned and started [31]. However, those approaches are not specific for urban projects and, at the best of our knowledge, interactive multicriteria methods have not been applied. Instead, other methods have been used; for example, [32] defines a weighted sum of an economic index and a social indicator while [33] develops a general procedure to generate efficient portfolios through a scatter search based metaheuristic. Moreover, in all the above cases, only quantitative evaluations for the different criteria have been considered.

Thus, the above literature review shows that, despite huge interest in developing multiobjective optimization approaches in the urban context, very little has been done in consideration with the timing of the projects and in the use of qualitative evaluations for the criteria. In the following we introduce our model that aims to fill such gaps.

\section{The proposed model}

In the following, we present our model to deal with the selection and the timing of urban planning projects. To help the reader, we also propose a small didactic example to explain the different elements of our model. It is important to highlight that the model allows dealing with a very large number of projects taking into account their performances with respect to considered criteria and their cost over different periods of time. 


\subsection{Notations and Data}

We consider a set of urban planning projects $A=\left\{a_{1}, \ldots, a_{j}, \ldots, a_{n}\right\}$ and a set of periods $T=\left\{t_{1}, \ldots, t_{t}, \ldots, t_{h}\right\}$. For each period $t \in T$ we have an available budget $b_{t} \in \mathbb{R}$. Each project $a_{j} \in A$ is characterized by an associated cost $\gamma_{j} \in \mathbb{R}$ and a duration $u_{j} \in \mathbb{R}$.

We have a family $D=\left\{D_{1}, \ldots, D_{h}\right\}$ of sets $D_{t}=\left\{d_{1, t}, \ldots, d_{m_{t}, t}\right\}$ containing strategic needs $d_{q t}$ to be satisfied in period $t \in T$. We denote with $s_{j q t}$ the contribution of a project $a_{j}$ to requirement $d_{q t} \in D_{t}$ with $t \in T$.

Each project $a_{j} \in A$ is evaluated on a set of criteria $G=\left\{g_{1}, \ldots, g_{i}, \ldots, g_{m}\right\}$. The evaluation of each project $a_{j}$ with respect to the criterion $g_{i} \in G$ is denoted with $g_{i}\left(a_{j}\right)$. These evaluations can be quantitative evaluations or qualitative evaluations. For the qualitative evaluations a set of qualitative levels $V_{i}$ for each criterion is defined and the evaluation $g_{i}\left(a_{j}\right)$ associates a qualitative level from $V_{i}$ to the project $a_{j} \in A$. The evaluations can also be specified in each period $t \in T$ and in this case they are denoted by $g_{i t}\left(a_{j}\right)$.

For the sake of simplicity, without the loss of generality, suppose that all criteria $g_{i}$ are of a gain type, such that the greater $g_{i t}\left(a_{j}\right)$ the better evaluation of the alternative $a_{j} \in A$ on the criterion $g_{i}$ in period $t$. We define, as proposed in [34], for each criterion $g_{i}$, a set $L_{i}$ of quality thresholds $l_{c}$ :

$$
L_{i}=\left\{l_{1, i}, \ldots, l_{c, i}, \ldots, l_{q, i}: l_{1, i}<l_{2, i}<\ldots<l_{q, i}\right\}
$$

permitting to define a set $\mathcal{C}_{i}$ consisting in $q(i)+1$ qualitative classes $C_{r i}$ :

$$
C_{i}=\left\{C_{1 i}, \ldots, C_{c i}, \ldots, C_{q(i)+1, i}\right\}
$$

such that the greater $c=1, \ldots, q(i)+1$, the more preferred is the project from the class $C_{c i}$. For the sake of simplicity and with a small abuse of the 
notation, in the following we shall denote classes $C_{c i}$ as $C_{c}$. The projects $a_{j} \in A$ belong to $C_{c} \in \mathcal{C}_{i}$ in period $t$ according to the following rules:

- $a_{j}$ is assigned to class $C_{1}$ in period $t$ if $g_{i t}\left(a_{j}\right)<l_{1, i, t}$;

- $a_{j}$ is assigned to class $C_{c}, c=2, \ldots, q$, in period $t$ if $l_{c-1, i, t} \leq g_{i t}\left(a_{j}\right)<$ $l_{c, i, t}$

- $a_{j}$ is assigned to class $C_{q(i)+1}$ in period $t$ if $g_{i t}\left(a_{j}\right) \geq l_{q+1, i}$

with $l_{c, i, t} \in L_{i}$ and $l_{1, i, t}<l_{2, i, t}<\ldots<l_{q, i, t}$ for all $t$.

\subsection{Variables and Objectives Function}

We define the following set of binary variables:

$$
x_{j t}= \begin{cases}1, & \text { if project } j \text { is planned to start in period } t \\ 0, & \text { otherwise }\end{cases}
$$

Therefore, we can denote a potential portfolio $P \subseteq A \times T$ with a vector $\mathbf{x}=\left[x_{11}, \ldots, x_{j t}, \ldots, x_{n h}\right]$.

Considering all the possible periods, for each potential portfolio $P \subseteq$ $A \times T$, each criterion $g_{i} \in G$ and each quality threshold level $l_{c, i, t} \in L_{i}$, we consider the set of projects $P_{c, i, t}$ attaining a certain threshold $l_{c, i, t}$ :

$$
P_{c, i, t}=\left\{a_{j} \in P: g_{i t}\left(a_{j}\right) \geq l_{c, i, t}\right\}
$$

Taking into account all the above elements, we formulate the decision problem as the following multi-objective optimization problem $\max F_{i, c}$ with:

$$
F_{i, c}=\sum_{t \in T}\left|P_{c, i, t}\right|, \quad \forall g_{i} \in G, \forall c \in C_{i}
$$


We can reformulate the objectives in terms of a binary variables introducing an indicator for each project $a_{j} \in A$, each criterion $g_{i} \in G$, each quality threshold $l_{c, i, t} \in L_{i}$ and each period $t \in T$ :

$$
h_{j, i, c, t}= \begin{cases}1, & \text { if } g_{i t}\left(a_{j}\right) \geq l_{c, i, t} \\ 0, & \text { otherwise }\end{cases}
$$

so that we have $\left|P_{c, i, t}\right|=\sum_{a_{j} \in A} x_{j t} h_{j, i, c, t}$ and the above mentioned multicriteria optimization problem can be formulated as follows:

$$
\max \sum_{a_{j} \in A, t \in T} x_{j t} h_{j, i, c, t} \quad \forall g_{i} \in G, \forall c \in C_{i}
$$

\subsection{Constraints}

When defining a problem concerning urban planning projects many constraints can be formulated. In our formulation we introduce three sets of constraints that may be further characterized on the basis of the particular application described.

The first set of constraints implies that each project can be scheduled at most once and only in one period:

$$
\sum_{t \in T} x_{i t} \leq 1, \quad \forall i \in I .
$$

The second set of constraints takes into account the limited financial resources available, in terms of budgets available in each period, i.e. $\forall t \in T$

$$
\sum_{a_{j} \in A} x_{j, t} \gamma_{j} \leq b_{t}+\sum_{\tau=1}^{t-1} b_{\tau}-\sum_{a_{j} \in A} \sum_{\tau=1}^{t-1} x_{j, t} \gamma_{j}
$$

This formulation implies that the budget not used in the previous periods can be moved forward and used in the following ones. Let us also highlight 
that a present value at an interest rate $\rho$ could be considered to take into account that the budget $b_{\tau}$ is referring to different years [35].

Thus, we have supposed that the construction cost of each project $\left(\gamma_{j}\right)$ does not depend on the time in which the project has started, considering the costs as given information assessed in the early stage of the process. Nevertheless, this assumption is rather strong and can be modelled in a more realistic way introducing a cost estimation. In the case of urban projects, several different indexes could be used. Therefore, the cost of project $a_{j}$ for each period $t \in T$ can be further specified as $\gamma_{j t}$. Alternatively, when dealing with the construction of new buildings, a so-called Tender Price is adopted to forecast the cost of constructing a building in the following years. To estimate those Tender Prices several methods can be used from the multiple regression analysis to the time series analysis (see [36] and references therein). Therefore, we denote with $\delta_{j t}$ a tender price associated with project $a_{j} \in A$ in period $t \in T$ so that we can reformulate the budget constraints (9) as:

$\forall t \in T$

$$
\sum_{a_{j} \in A} x_{j t} \gamma_{j} \delta_{j t} \leq b_{t}+\sum_{\tau=1}^{t-1} b_{\tau}-\sum_{a_{j} \in A} \sum_{\tau=1}^{t-1} x_{j, t} \gamma_{j} \delta_{j \tau}
$$

Finally, the third set of constraints is related to the strategic needs to meet, forcing that the contribution of the selected projects will cover the requirements of the organizations:

$\forall t \in T, \forall q \in D$,

$$
\sum_{a_{j} \in A} \sum_{\tau=1}^{t-u_{j}} x_{j t} s_{j q t} \geq \sum_{t=1}^{t} d_{q t}
$$

We also point out that uncertainty in the performances of urban projects with respect to the considered criteria can be taken into account. If we are 
Table 1: Cost $\gamma_{j t}$ of each project $a_{j}$ in each period $t$.

\begin{tabular}{|l||c|c|}
\hline Project & First period & Second period \\
\hline$a_{1}$ & 19 & 45 \\
$a_{2}$ & 36 & 46 \\
\hline
\end{tabular}

dealing with quantitative information we could adopt the same approach followed by [37] in which the expected value of the evaluation in a given period is computed using the probability of different states of nature. For the qualitative evaluations, different methods in the literature have been proposed to calculate a cardinal probabilistic evaluation of each project (see e.g., [38]). Analogously, uncertainty could be included in the definition of the qualitative thresholds.

\subsection{Didactic Example}

Let us introduce a didactic example in order to show how our model can be formulated. Let us suppose to have two projects $A=\left\{a_{1}, a_{2}\right\}$ that can be scheduled to start in two periods $T=\{1,2\}$. For the sake of simplicity, let us suppose that both the projects are related to the refurbishment of two different buildings. We have an available budget in the first period equals to $b_{1}=50$ and in the second period equals to $b_{2}=30$. In Table 1 we report the costs of each project for each period.

We have a single strategic need to meet. In particular, let us suppose that we have a requirement to refurbish at least $200 \mathrm{~m}^{2}$ in each period. Project $a_{1}$ completes refurbishment of $300 \mathrm{~m}^{2}$ independently of the period in which it is planned. Project $a_{2}$, if scheduled in the first period completes only $150 \mathrm{~m}^{2}$ of refurbishment, while in the second period covers $200 \mathrm{~m}^{2}$. Therefore, we have requirements i.e. $d_{11}=d_{12}=$ total area of refurbishment 
Table 2: Evaluation of each project $a_{j}$ on each criterion $g_{i t}$ in each period $t$

\begin{tabular}{|c||c|c|}
\hline & First period & Second period \\
\hline$g_{1}$ & 10 & 5 \\
$g_{2}$ & $\mathrm{~L}$ & $\mathrm{~V}-\mathrm{H}$ \\
\hline
\end{tabular}

(a) Project $a_{1}$

\begin{tabular}{|c||c|c|}
\hline & First period & Second period \\
\hline$g_{1}$ & 4 & 4 \\
$g_{2}$ & $\mathrm{M}$ & $\mathrm{H}$ \\
\hline
\end{tabular}

(b) Project $a_{2}$

Table 3: Quality thresholds $l_{c, i, t}$ for each criterion $g_{i}$ and each period $t$

\begin{tabular}{|c||c|c|}
\hline & First period & Second period \\
\hline$g_{1}$ & 5 & 5 \\
$g_{2}$ & $\mathrm{M}$ & $\mathrm{M}$ \\
\hline
\end{tabular}

(a) First quality threshold $l_{1, i, t}$

\begin{tabular}{|c||c|c|}
\hline & First period & Second period \\
\hline$g_{1}$ & 10 & 15 \\
$g_{2}$ & $\mathrm{~V}-\mathrm{H}$ & $\mathrm{V}-\mathrm{H}$ \\
\hline
\end{tabular}

(b) Second quality thresholds $l_{2, i, t}$

$\geq 200 \mathrm{~m}^{2}$ (both for period 1 and period 2), while the contribution of the projects is $s_{111}=s_{112}=300$ while $s_{211}=150$ and $s_{212}=200$. Let us underline that these constraints are extremely simplified in order to make as easy as possible the understanding of the model.

Each project has been evaluated on two criteria $G=\left\{g_{1}, g_{2}\right\}$. The first criterion, let us describe it as thousands $m^{2}$ refurbished, can be expressed as a piece of quantitative information in the range $[0,10]$ while the second criterion, how sustainable are the interventions, is evaluated on a 4-level likert scale: Low (L), Medium (M), High (H), Very High (VH). In Table 2 we report the contribution of each project for each criterion in each period.

In Table 3 we report the quality thresholds defined for each criterion in each period. We suppose that for each criterion we have two possible quality thresholds levels $L_{i}=\left\{l_{1, i, 1}, l_{2, i, 1}, l_{1, i, 2}, l_{2, i, 2}\right\}, i=1, \ldots, n$.

At this point we can compute the binary variable $h_{j, i, c, t}$ as in Table 4. 
Table 4: Binary variables $h_{j, i, c, t}$ for each project $a_{j}$, each satisfaction level $l_{c, i, t}$ and each period $t$

\begin{tabular}{|l||c|c|}
\hline & First period & Second period \\
\hline$l_{1,1}$ & 1 & 1 \\
$l_{2,1}$ & 1 & 0 \\
\hline
\end{tabular}

(a) Project $a_{1}$, criterion $g_{1}$

\begin{tabular}{|l||c|c|}
\hline & First period & Second period \\
\hline$l_{1,2}$ & 0 & 1 \\
$l_{2,2}$ & 0 & 1 \\
\hline
\end{tabular}

(c) Project $a_{1}$, criterion $g_{2}$

\begin{tabular}{|l||c|c|}
\hline & First period & Second period \\
\hline$l_{1,1}$ & 0 & 0 \\
$l_{2,1}$ & 0 & 0 \\
\hline
\end{tabular}

(b) Project $a_{2}$, criterion $g_{1}$

\begin{tabular}{|c||c|c|}
\hline & First period & Second period \\
\hline$l_{1,2}$ & 1 & 1 \\
$l_{2,2}$ & 0 & 0 \\
\hline
\end{tabular}

(d) Project $a_{2}$, criterion $g_{2}$

On the basis of constraints (8) only eight portfolios are feasible, i.e. including one project or both the projects with each of them scheduled in one of the two periods. Let us underline that, for the sake of simplicity, in this example we did not take into account the duration $u_{j}$ of each project.

Among those, on the basis of the requirement constraint (11), we need to discard the portfolio composed only by project $a_{2}$ scheduled in the first period because it does not satisfy the requirement of delivering at least 200 $m^{2}$ of refurbishment. Similarly, on the basis of the budget constraints, some portfolios are not feasible. While there is enough budget to implement every single project in each period, only a pair of projects can be implemented together, i.e. project $a_{1}$ in the first period and project $a_{2}$ in the second period. In the following, for each of the feasible portfolios we can calculate the objective function as follows:

- Portfolio $P_{1}=\left\{x_{11}\right\}$, where project $a_{1}$ is planned to start in the first period. This portfolio presents 2 contributions that are above or equal 
to the defined quality thresholds, i.e.

$$
\begin{aligned}
& g_{11}\left(a_{1}\right) \geq l_{1,1,1}, \\
& g_{11}\left(a_{1}\right) \geq l_{2,1,1} .
\end{aligned}
$$

- Portfolio $P_{2}=\left\{x_{12}\right\}$, where project $a_{1}$ is planned to start in the second period. This portfolio presents 3 contributions that are above or equal the defined quality thresholds, i.e.

$$
\begin{aligned}
& g_{12}\left(a_{1}\right) \geq l_{1,1,2}, \\
& g_{22}\left(a_{1}\right) \geq l_{1,2,2}, \\
& g_{22}\left(a_{1}\right) \geq l_{2,2,2} .
\end{aligned}
$$

- Portfolio $P_{3}=\left\{x_{22}\right\}$, where project $a_{2}$ is planned to start in the second period. This portfolio presents 1 contribution that is above or equal the defined quality thresholds, i.e.

$$
g_{22}\left(a_{2}\right) \geq l_{1,2,2}
$$

- Portfolio $P_{4}=\left\{x_{11}, x_{22}\right\}$, where project $a_{1}$ is planned to start in the first period and project $a_{2}$ is planned to start in the second period. This portfolio presents 3 contributions that are above or equal the defined quality thresholds, i.e.

$$
\begin{aligned}
& g_{11}\left(a_{1}\right) \geq l_{1,1,1}, \\
& g_{11}\left(a_{1}\right) \geq l_{2,1,1}, \\
& g_{22}\left(a_{2}\right) \geq l_{1,2,2} .
\end{aligned}
$$


Then, we can define the functions that assigns to each portfolio $P$ the value $F(P, i, c)=\sum_{t \in T}\left|P_{c, i, t}\right|$ with $c=1,2$ and $t=1,2$, as follows:

- Portfolio $P_{1}$ :

$$
F(1,1)=1, F(1,2)=1, F(2,1)=0, F(2,2)=0,
$$

- Portfolio $P_{2}$ :

$$
F(1,1)=1, F(1,2)=0, F(2,1)=1, F(2,2)=1
$$

- Portfolio $P_{3}$ :

$$
F(1,1)=0, F(1,2)=0, F(2,1)=1, F(2,2)=0
$$

- Portfolio $P_{4}$ :

$$
F(1,1)=1, F(1,2)=1, F(2,1)=1, F(2,2)=0
$$

\section{Method}

Several methods could be applied to search for the most preferred portfolio. The complexity of these problems, and the challenging computational efforts necessary to find solutions for those problems, often implies the adoption of metaheuristic approaches [39]. Among those, evolutionary algorithms could be applied with the aim of computing the whole Pareto front including all the efficient solutions [17]. However, even if they can tackle big instances (see e.g. [40] [41, 42]) for a variety of applications (e.g. [43]), their use still implies that the DM is able to choose the most preferred solutions among the possible overwhelming number of solutions in the Pareto front a posteriori. Indeed, for the complexity of the problem described and the necessity of 
discussing the characteristics of the portfolio with the various stakeholders, such approaches seem to create a profusion of information supplied by the multiobjective optimization procedure that it may be difficult to handle for the stakeholders [44]. Therefore, it seems that an Interactive Multi-objective Optimization (IMO) methodology [45] could provide a more appropriate approach. Usually, in an interactive approach the computation phase, where an optimization problem is solved, is followed by a dialogue phase where the obtained portfolio is presented to the DM and his feedback becomes the preference information that drives the procedure in the next iteration of the method. The interactive methods aim to reach the most preferred solution of the Pareto front of the problem by means of an algorithmic procedure directed by the preferences of the DMs [46]. Since a single solution is searched for and it is not necessary to discover the whole Pareto front, the computational complexity of multiobjective portfolio problems is relatively low and even large instances can be successfully handled. In addition to that, the DMs do not have to choose a solution a posteriori after the construction of the whole Pareto front that can be very difficult from a cognitive point of view, especially when dealing with complex problems as it is the case for the urban planning problems [45].

Therefore, we can list the following benefits:

- IMO methods can deal with portfolio problems for defining a selection of projects [34].

- Several interactions can be conducted with the DM and visualization tools can be used in order to help his understanding of how the portfolio is evolving in the time [47].

- IMO methods allow to handle several criteria even with qualitative 
evaluations. Moreover, several different imprecise evaluations can be handled through the repetition of the interaction with the DM [45].

- Several scenarios can be simulated and robustness analysis can be used to verify the goodness of such solutions [48].

- Several actors can be involved and the IMO methods can even be used in a focus group environment [49].

Among the many IMO methods proposed in the literature, we choose a method called IMO-DRSA [50] but, any other IMO method can be applied as well. In the IMO-DRSA an interactive procedure is integrated into a multiobjective optimization procedure with the use of the Dominance Based Rough Set Approach (DRSA) (see, e.g., [51, 52]). In the computation phase some portfolios are developed thanks to the resolution of an optimization problem. In the dialogue phase those portfolios are presented to the DM and he is asked to select one if he is convinced that it is completely satisfactory. In this case the procedure ends. Otherwise, the DM is asked to indicate a set of relatively good portfolios in the list, so that a binary partition into classes "good" and "others" of the list of proposed portfolios can be generated. This interactive method seems to be particularly appropriate to our case given that all the performances have been already transformed in qualitative ordinal evaluations by means of suitable qualitative levels.

More in detail, for each criterion $g_{i} \in G$ and for each satisfaction level represented by the class $c \in C_{i}$, one determines a portfolio that maximizes $F_{i, c}$ i.e., the number of projects that attain value at least $l_{c, i, t}$ on criterion $g_{i}$ considering the whole set of periods $T$. In this way, in the computation phase, we find a set of portfolios that maximize individually each objective $F_{i, c}$ that we show to the DM. The sets presented to the DM in the following 
iterations of the interactive procedure can be determined analogously. For a more detailed description of the method see [34]. Let us highlight that those optimization problems can be solved very quickly with any commercial software.

In the dialogue phase the DM indicates what are the portfolios that are considered good and the others and a set of decision rules with the following syntax can be induced

"if $F_{i_{1}, c_{1}}(\mathbf{x}) \geq \alpha_{i_{1}, c_{1}}$ and $\ldots$ and $F_{i_{q}, c_{q}}(\mathbf{x}) \geq \alpha_{i_{q}, c_{q}}$, then portfolio $\mathbf{x}$ is good",

meaning that a portfolio can be considered good only if it contains a number of projects in class $C_{c_{i_{p}}+1, i_{p}}$ or better for each criterion $g_{i_{p}}, p=$ $1, \ldots, q$ considered in the rule. Those rules are taken into consideration in the subsequent computation phase where the optimization model is solved again with the addition of constraints representing the conditions in the "if" part of the selected rule.

\subsection{DRSA method for the Didactic Example}

Let us present in Table 5 the set of portfolios introduced in Section 3 in terms of the $F_{i, c}$. For example, $F_{1,1}$ is related to the number of projects $a_{j}$ such that $g_{1 t}\left(a_{j}\right) \geq l_{1,1, t}$ in any period $t$ subject to the constraints of the original model and so on.

In the computation phase we have two criteria and two satisfaction levels, meaning that we optimise four different objective functions and we obtain four non dominated portfolios. For example, optimizing criterion $g_{1}$ at its first level $l_{1,1, t}$ the optimal portfolio is one among portfolios $P_{1}, P_{2}$ and $P_{4}$. Instead, when optimizing criterion $g_{2}$ at its second level $l_{2,2, t}, P_{2}$ is the optimal portfolio.

In the dialogue phase the DM will indicate what are the portfolios that he 
Table 5: Portfolios in the didactic example represented in terms of the $F_{i, c}$.

\begin{tabular}{|c|c|c|c|c|}
\hline Portfolio & $F_{1,1}$ & $F_{1,2}$ & $F_{2,1}$ & $F_{2,2}$ \\
\hline$P_{1}$ & 1 & 0 & 1 & 0 \\
$P_{2}$ & 1 & 1 & 0 & 1 \\
$P_{3}$ & 0 & 1 & 0 & 0 \\
$P_{4}$ & 1 & 1 & 1 & 0 \\
\hline
\end{tabular}

considers "good". Let us suppose that the DM indicates as good portfolios $P_{3}$ and $P_{4}$. Both the portfolios are characterized by having $F_{2,1} \geq 1$. This will be assumed as a rule extracted by the preferences expressed by the DM and it will be included in the next computation phase in the optimization problem.

\section{A case study: selection of projects to include in a University Master Plan}

The redaction of a Master Plan of an organization is one of the most challenging points that an organization needs to face. This is even more true when the Master Plan regards a University that needs to forecast its future needs in an uncertain historical period. In addition to that, many universities are located in city centres and they need to integrate their facilities in the local urban environment satisfying strict requirements provided by the local authorities.

The Master Plan of the University of Portsmouth was released in 2016 and indicated a series of interventions that the University aimed to complete in order to achieve a series of objectives and meet some strategic requirements. However, the Master Plan was thought and published in a 
period where the economic situation was more stable and before the results of the Brexit referendum increased the level of uncertainty. Therefore, it emerged the necessity of revising the plan on the assumption that the costs of the projects increased, the budget decreased and the requirements to fulfill changed. Moreover, different stakeholders may be involved including students, staff, local community and local authorities. Thus, in our analysis, we propose to use our model to analyse how those changes can be taken into account, simulating possible different scenarios that, in case of their occurrence, the University should promptly and properly handle.

The Master Plan of the University is a public document that can be retrieved on the University website. In our showcase for our model we considered these documents together with other information that can be retrieved on the internet. In addition, one of the authors has expertise in urban planning and has provided further elaboration of the data provided in the analysis.

In the following we report:

- The objectives pursued by the Master Plan and the criteria used to evaluate the different possible future intervention;

- The interventions that have been included in the Master Plan and the different scenarios that we want to analyse;

- The model and the method implemented for these particular applications.

\subsection{Objectives}

As criteria of our problem we assume the objectives listed in the Master Plan. We can collect these objectives in three macro-categories. 
1. Development of University campus

This category includes objectives that describe how the different projects can enhance different aspects concerning the quality of the University buildings. Therefore, the criteria listed are:

(a) Increasing the quality of the University Estate in terms of spaces: | notation: $g_{1} \cdot \mid$

This criterion describes how much the interventions will improve the quality of the buildings. A 4-level qualitative scale is adopted as follows:

- Low (L): major repair or upgrades needed for a below average quality building.

- Medium (M): major repair or upgrades needed, for an average quality building;

- Good (G): minor repairs as routine maintenance, for an above average quality building;

- Very Good (VG): brand-new buildings to build;

(b) Improving the coherence of the spaces:

| notation: $g_{2}$ |

This criterion compares the compatibility of the current characteristics of a building with the new planned uses. The 4-level qualitative scale adopted is:

- Low (L): the building no longer fits for purpose;

- Medium (M): building could have a new lease of life with major remodelling;

- Good (G): building needs reorganization of internal spaces; 
- Very Good (VG): building fits for purpose and contribute positively to the campus;

(c) Enhancing the sense of identity and arrival:

| notation: $g_{3}$ |

This criterion measures the quality of the improvement in terms of aggregations spaces created by the listed projects. A 5-level qualitative scale that classifies the degree of improvement is adopted; in addition to the 4-level qualitative scale used for criterion 1.a the additional level "no improvement (NO)" is introduced.

2. Enhancement of students and staff experience

The aim is to provide facilities that promote enjoyment and engagement within the wide community of learning to satisfy students and staff needs and expectations.

(a) Creation of innovative teaching and learning spaces:

| notation: $g_{4}$ |

This criterion evaluates the increase of innovative spaces dedicated to advanced teaching methodologies; a 5-level qualitative scale as in criterion 1c is adopted.

(b) Enrichment of the spaces dedicated to research activities: | notation: $g_{5}$ |

This criterion measures the number of the new researches that can operate in the new facilities.

(c) Improving of social learning spaces equipment:

| notation: $g_{6} ; \mid$ 
This criterion describes the quality of the new spaces added in terms of social learning spaces. A 5-level qualitative scale as in criterion $1 \mathrm{c}$ is adopted.

3. Sustainability of the interventions

The University is located in a city centre campus and plays a central role in the life of the city. The University is keen to maintain an excellent relationship with the city and its community ensuring benefits to both University and the whole city. In addition, the sustainable creation of a coherent network of routes and services can improve the urban environment of the city.

(a) Increasing Green and Student mobility:

| notation: $g_{7}$ |

The University aims to reduce reliance on private cars and incentives for sustainable forms of transport (cycling, walking, public transport). The criterion measures how much the projects help to reach a green mobility for staff and students. A 5-level qualitative scale as in criterion 1c is adopted.

(b) Enhancement of community social engagement:

| notation: $g_{8}$ |

The University aims to maximise the opportunities to create a social sustainable environment improving the social opportunities of the whole community. A 5-level qualitative scale as in criterion $1 \mathrm{c}$ is adopted.

(c) Increasing the economic benefits:

| notation: $g_{9}$ | 
The different interventions will create a new number of jobs in the long term, developing a new and sustainable economic perspective for the whole community requests an increase in the number of employees. This criterion evaluates the creation of new jobs linked to the new projects.

\subsection{Proposed Interventions and Requirements}

In this subsection we present a list of the projects included in the Master Plan. The University planned to refurbish several existent buildings and external areas, to create new spaces for teaching and leisure activities and to build brand new buildings. A brief description of each project can be retrieved in the Appendix. In Table 6 we report the contribution of the potential set of sixteen projects $A=\left\{a_{1}, \ldots, a_{16}\right\}$ included in the Master Plan, for each element of the set of the nine criteria $G=\left\{g_{1}, \ldots, g_{9}\right\}$. Although the evaluation $g_{i t}\left(a_{j}\right)$ could be specified for every period, in this case the evaluations have been considered equivalent in every period $t \in T$. Similarly, we consider the quality thresholds as independent of the time and we suppose to have three qualitative levels $L_{i}$ for each criterion $g_{i}$ as reported in Table 7 so that $l_{c, i, t}=l_{c, i} \forall t \in T$.

Although the current Master Plan of the University was covering only 15 years, some of the benefits of the projects will extend further in the time. Consequently, the authors decided to consider a horizon planning of 20 years, i.e. $T=\{1, \ldots, 20\}$. In order to simulate the uncertainty concerning the budget available, we consider three feasible amounts of budget available $B=\left\{b_{1}, b_{2}, b_{3}\right\}$ distributed in the planning period of 20 years. Given that funds to the University are allocated every 5 years, we divide the budget allocated every five-year in a cyclic pattern distributing the majority of the 
Table 6: Performances $g_{i}\left(a_{j}\right)$ for every project $a_{j}$ and for every criterion $g_{i}$.

\begin{tabular}{cccccccccc}
\hline \hline Projects & $g_{1}$ & $g_{2}$ & $g_{3}$ & $g_{4}$ & $g_{5}$ & $g_{6}$ & $g_{7}$ & $g_{8}$ & $g_{9}$ \\
\hline \hline$a_{1}$ & $\mathrm{VG}$ & $\mathrm{VG}$ & $\mathrm{VH}$ & $\mathrm{H}$ & 634 & $\mathrm{~L}$ & $\mathrm{NO}$ & $\mathrm{H}$ & 1008 \\
$a_{2}$ & $\mathrm{M}$ & $\mathrm{M}$ & $\mathrm{NO}$ & $\mathrm{NO}$ & 0 & $\mathrm{NO}$ & $\mathrm{M}$ & $\mathrm{L}$ & 0 \\
$a_{3}$ & $\mathrm{VG}$ & $\mathrm{VG}$ & $\mathrm{M}$ & $\mathrm{NO}$ & 0 & $\mathrm{VH}$ & $\mathrm{NO}$ & $\mathrm{H}$ & 276 \\
$a_{4}$ & $\mathrm{VG}$ & $\mathrm{VG}$ & $\mathrm{L}$ & $\mathrm{M}$ & 40 & $\mathrm{M}$ & $\mathrm{NO}$ & $\mathrm{M}$ & 63 \\
$a_{5}$ & $\mathrm{~L}$ & $\mathrm{G}$ & $\mathrm{H}$ & $\mathrm{NO}$ & 0 & $\mathrm{~L}$ & $\mathrm{H}$ & $\mathrm{H}$ & 0 \\
$a_{6}$ & $\mathrm{M}$ & $\mathrm{L}$ & $\mathrm{M}$ & $\mathrm{H}$ & 127 & $\mathrm{NO}$ & $\mathrm{NO}$ & $\mathrm{L}$ & 202 \\
$a_{7}$ & $\mathrm{M}$ & $\mathrm{M}$ & $\mathrm{M}$ & $\mathrm{L}$ & 0 & $\mathrm{NO}$ & $\mathrm{NO}$ & $\mathrm{L}$ & 0 \\
$a_{8}$ & $\mathrm{M}$ & $\mathrm{M}$ & $\mathrm{L}$ & $\mathrm{NO}$ & 0 & $\mathrm{NO}$ & $\mathrm{L}$ & $\mathrm{M}$ & 0 \\
$a_{9}$ & $\mathrm{~L}$ & $\mathrm{~L}$ & $\mathrm{VH}$ & $\mathrm{NO}$ & 0 & $\mathrm{VH}$ & $\mathrm{L}$ & $\mathrm{L}$ & 64 \\
$a_{10}$ & $\mathrm{M}$ & $\mathrm{L}$ & $\mathrm{M}$ & $\mathrm{NO}$ & 214 & $\mathrm{~V}$ & $\mathrm{NO}$ & $\mathrm{NO}$ & 340 \\
$a_{11}$ & $\mathrm{M}$ & $\mathrm{L}$ & $\mathrm{H}$ & $\mathrm{H}$ & 236 & $\mathrm{~L}$ & $\mathrm{M}$ & $\mathrm{M}$ & 375 \\
$a_{12}$ & $\mathrm{M}$ & $\mathrm{L}$ & $\mathrm{H}$ & $\mathrm{H}$ & 0 & $\mathrm{~L}$ & $\mathrm{~L}$ & $\mathrm{~L}$ & 0 \\
$a_{13}$ & $\mathrm{~L}$ & $\mathrm{G}$ & $\mathrm{H}$ & $\mathrm{NO}$ & 0 & $\mathrm{~L}$ & $\mathrm{H}$ & $\mathrm{H}$ & 0 \\
$a_{14}$ & $\mathrm{VG}$ & $\mathrm{VG}$ & $\mathrm{L}$ & $\mathrm{M}$ & 178 & $\mathrm{M}$ & $\mathrm{M}$ & $\mathrm{M}$ & 284 \\
$a_{15}$ & $\mathrm{M}$ & $\mathrm{L}$ & $\mathrm{M}$ & $\mathrm{M}$ & 119 & $\mathrm{~L}$ & $\mathrm{~L}$ & $\mathrm{M}$ & 189 \\
$a_{16}$ & $\mathrm{M}$ & $\mathrm{L}$ & $\mathrm{VH}$ & $\mathrm{NO}$ & 0 & $\mathrm{~L}$ & $\mathrm{M}$ & $\mathrm{H}$ & 315 \\
\hline \hline
\end{tabular}

amount at the beginning of the five-year pattern (for a detailed distribution of the budget $b_{t}$ in the 20 years see the Appendix). On the unused capital we computed interests according to the guidelines published in the UK's Treasury Green Book at a rate $\rho$ equal to 0.035 [53]. The cost $\gamma_{j}$ of each project $a_{j} \in A$ is reported in Table 8 . Moreover, we have introduced a Tender Price index, $\delta_{j t}$ that takes into account the variation of the building cost during the time [54]. It's important to highlight that the contribution 
Table 7: Quality thresholds $l_{c, i}$ for each criterion $g_{i}$.

\begin{tabular}{rccc}
\hline \hline Criterion & $l_{1, i}$ & $l_{2, i}$ & $l_{3, i}$ \\
\hline \hline$g_{1}$ & $\mathrm{M}$ & $\mathrm{H}$ & $\mathrm{VH}$ \\
$g_{2}$ & $\mathrm{M}$ & $\mathrm{H}$ & $\mathrm{VH}$ \\
$g_{3}$ & $\mathrm{~L}$ & $\mathrm{M}$ & $\mathrm{H}$ \\
$g_{4}$ & $\mathrm{M}$ & $\mathrm{H}$ & $\mathrm{VH}$ \\
$g_{5}$ & 100 & 200 & 350 \\
$g_{6}$ & $\mathrm{~L}$ & $\mathrm{M}$ & $\mathrm{VH}$ \\
$g_{7}$ & $\mathrm{M}$ & $\mathrm{H}$ & $\mathrm{VH}$ \\
$g_{8}$ & $\mathrm{~L}$ & $\mathrm{M}$ & $\mathrm{H}$ \\
$g_{9}$ & 100 & 300 & 500 \\
\hline \hline
\end{tabular}

of each project is taken into account only after the complete realization of the building intervention. At the same time, the necessary budget for each project needs to be available when the project is scheduled. Therefore, in Table 8 for each project $a_{j}$ we report also its duration $u_{j}$.

In addition to the budget constraint, the University has to satisfy requirements related to the space dedicated to teaching and leisure activities. In this initial planning phase this is assumed to be the only requirement. In particular, the available space is related to the expected growth of students in the next year. The space that will be generated by each project $a_{j} \in A$ is indicated with $s_{j 1}$ and it is reported in Table 8. The area generated is independent of the period in which the project will be scheduled.

The University stated that expected a five-year student growth of $10 \%$ by 2020. However, given the high level of uncertainty derived from several factors (i.e. the political uncertainty), it seems more appropriate to consider 
Table 8: For each project $a_{j} \in A$ we have: Cost $\gamma_{j}$, Refurbished Area $s_{j 1}$ and duration $u_{j}$

\begin{tabular}{cccc}
\hline \hline Cost & $\begin{array}{c}\text { Refurbished Area } \\
\left(m^{2}\right)\end{array}$ & $\begin{array}{c}\text { Duration } \\
\text { (Years })\end{array}$ \\
\hline \hline$a_{1}$ & 90.5 & 16000 & 4 \\
$a_{2}$ & 0.5 & 0 & 1 \\
$a_{3}$ & 47.0 & 7500 & 3 \\
$a_{4}$ & 6.0 & 1000 & 1 \\
$a_{5}$ & 5.0 & 0 & 1 \\
$a_{6}$ & 59.5 & 3500 & 3 \\
$a_{7}$ & 6.0 & 0 & 1 \\
$a_{8}$ & 0.5 & 0 & 1 \\
$a_{9}$ & 31.0 & 2000 & 2 \\
$a_{10}$ & 28.5 & 5500 & 2 \\
$a_{11}$ & 88.5 & 6000 & 4 \\
$a_{12}$ & 30.0 & 0 & 3 \\
$a_{13}$ & 1.5 & 0 & 1 \\
$a_{14}$ & 25.0 & 4500 & 2 \\
$a_{15}$ & 34.5 & 3000 & 2 \\
$a_{16}$ & 73.5 & 9000 & \\
\hline \hline & & & \\
\hline
\end{tabular}

also a smaller growth of number of the students. Thus, in relation to the space standards, we have followed the AUDE space standards (2010) which are based on different parameters linked to volume of activity, hours of delivery, and the ratio of staff and students. These guidelines have been applied in the last few years by several UK Universities (see e.g. U.C.L., 
Table 9: Possible scenarios $S_{r, b}$

\begin{tabular}{|c|c|c|}
\hline Scenario & Growth & Budget available \\
\hline \hline$S_{1,1}$ & $10 \%$ & 530 \\
\hline$S_{1,2}$ & $7,5 \%$ & 380 \\
\hline$S_{1,3}$ & $5 \%$ & 280 \\
\hline$S_{2,1}$ & $10 \%$ & 530 \\
\hline$S_{2,2}$ & $7,5 \%$ & 380 \\
\hline$S_{2,3}$ & $5 \%$ & 280 \\
\hline$S_{3,1}$ & $10 \%$ & 530 \\
\hline$S_{3,2}$ & $7,5 \%$ & 380 \\
\hline$S_{3,3}$ & $5 \%$ & 280 \\
\hline
\end{tabular}

University of Stirling, and University of Brighton). Therefore, we formulate three scenarios $R=\left\{r_{1}, r_{2}, r_{3}\right\}$ based on different growth forecasting for the number of students. In particular, we consider a five-year number of student growth of $10 \%, 7.5 \%$ and $5 \%$, therefore the requirements related to the refurbished area will increase every five years of 5\%, $7.5 \%$ and $10 \%$ for each scenario, respectively.

Thus, combining the budgets available and the minimum requirements needed, we obtain a total of 9 scenarios that we denote by $S_{r, b}$ where $r$ refers to the possible student growth values, and $b$ to the budget available. In Table 9 we present those possible scenarios.

\subsection{Model Application}

We solved for each criterion, for each quality threshold and for each scenario described in the previous section, the model introduced in Sec- 
Table 10: Composition of the portfolios obtained optimizing for each scenario $S_{r, b}$ each objective

\begin{tabular}{|c|c|c|c|}
\hline Scenario & \# Min & \# Max & \# Portfolios \\
\hline \hline$S_{1,1}$ & 13 & 15 & 24 \\
\hline$S_{1,2}$ & \multicolumn{3}{|c|}{-} \\
\hline$S_{1,3}$ & \multicolumn{3}{|c|}{} \\
\hline$S_{2,1}$ & 10 & 15 & 25 \\
\hline$S_{2,2}$ & 8 & 13 & 21 \\
\hline$S_{2,3}$ & & - \\
\hline$S_{3,1}$ & 11 & 15 & 25 \\
\hline$S_{3,2}$ & 9 & 13 & 25 \\
\hline$S_{3,3}$ & 10 & 11 & 23 \\
\hline
\end{tabular}

tion 3. Therefore, the problem becomes a single objective binary problem, that, even for big instances, can be easily solved with exact algorithms and a relatively low computational complexity [55]. In our case, we used the commercial software CPLEX, even if any other solver could be used. The computational times were less than 5 seconds for each instance, on a standard personal computer ${ }^{1}$.

In Table 10 we report for each scenario:

- \# Min, i.e. the number of projects in the portfolio with the smallest number of projects;

\footnotetext{
${ }^{1}$ Let us highlight that in order to test if the model could be solved for bigger instances we developed some random instances with up to 100 projects. The computational time was still very small, with an average of about six seconds even for the biggest instances.
} 
- \# Max, i.e.the number of projects in the portfolio with the largest number of projects;

- \#Portfolio, i.e. the number of different portfolios, i.e. composed of different projects or with the same projects but scheduled in different periods.

Let us discuss the following remarks:

- Some of the scenarios did not produce a feasible solution given that the imposed constraints were too restrictive considering the combination of the allocated budget and the requirements to satisfy in terms of area needed for the increasing number of students. In the Table the symbol " - " indicates the case in which a feasible solution could not be obtained;

- When the budget is increased, a larger number of projects can be selected (up to 15);

- When requirements related to the growth of the number of students are weakened a very large variety of portfolios can be retrieved;

- Scenario $S_{2,2}$ presents a great diversity in the number of projects included in the portfolios depending on the objective that we are optimizing (from 8 projects to 13 projects).

Let us underline that when it is not possible obtaining feasible portfolios we could interact with the DM in order to relax the set of constraints after discussing with him the relevance of each of them. 


\subsection{Application of the method}

In order to show how the method introduced in Section 4 can support the DM to choose the most preferred portfolios obtained from our model, we describe its implementation for one of the scenarios. To reflect the latest trend and policies indicated by the University, the authors decided to apply the method described above to the scenario $S_{2,2}$ which represents a good trade-off in terms of precautionary hypothesis: in fact, it combines a reduced growth in the number of students and a smaller budget available with respect to the scenario considered in the initial University strategy. To implement the method, the portfolios obtained in Scenario $S_{2,2}$ were represented with a visual timing distribution in order that it would be easier to understand what are the projects selected and when they will be scheduled. For example, in Figure 1 we report the portfolio obtained optimizing objective $P_{2,2}$. We can see that for this portfolio:

- Project $a_{10}$ and $a_{14}$ are scheduled to start in year 3 ;

- Project $a_{3}$ is scheduled to start in year 7;

- Project $a_{1}$ is scheduled to start in year 11 ;

- Project $a_{6}$ is scheduled to start in year 17 ;

- Project $a_{9}$ is scheduled to start in year 18 ;

- Projects $a_{5}, a_{12}$ and $a_{13}$ are scheduled to start in year 20 .

In Tables 11, 12 and 13 we report the portfolios obtained from the optimization of the $P_{c, i}$ represented in term of the values of $F_{i, c}$ (let us underline that we split the portfolios in three tables for the sake of the space). We have 9 criteria and 3 qualitative levels for each criterion so that we obtain a 


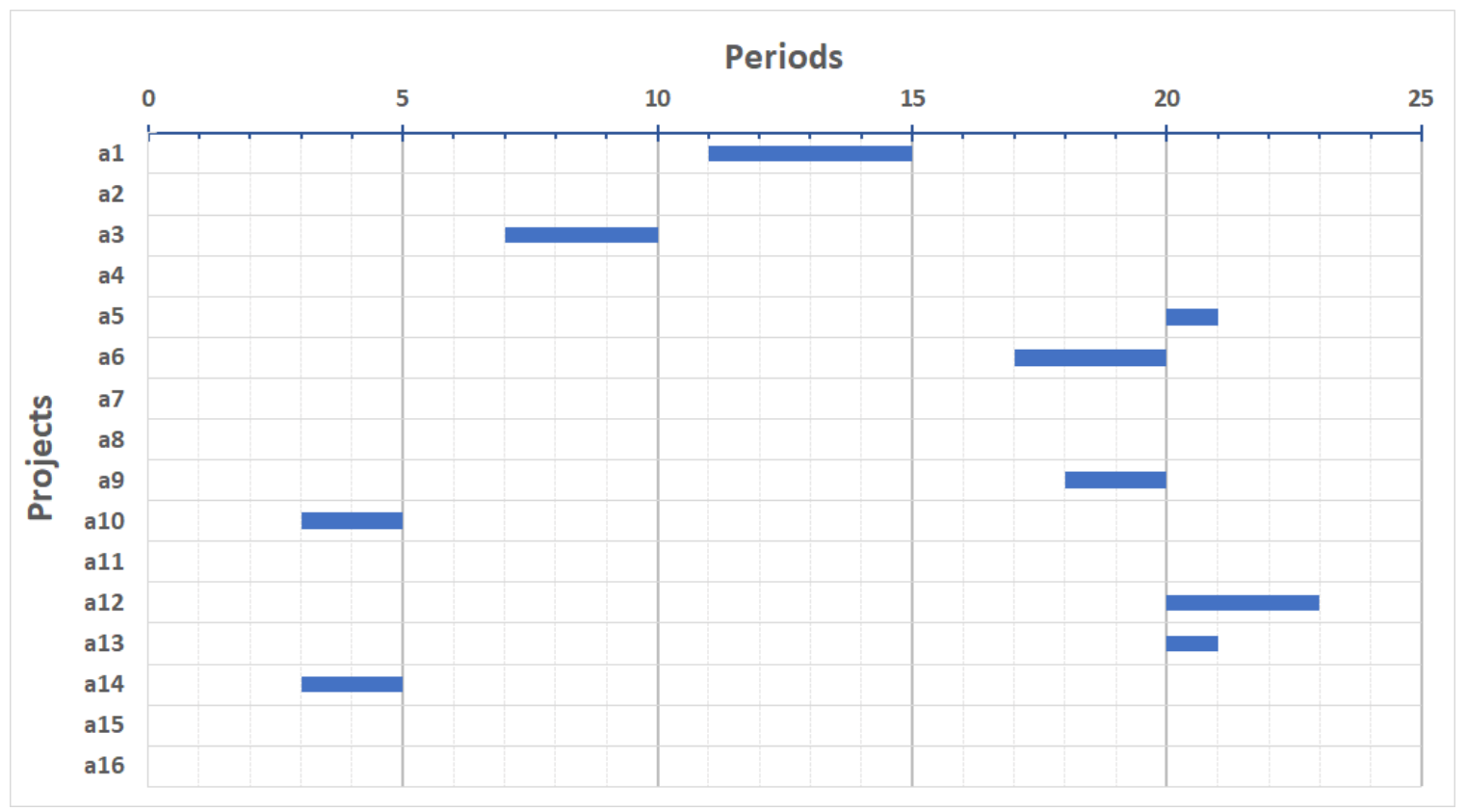

Figure 1: Selection and timing of the projects obtained optimizing $P_{2,2}$ for Scenario $S_{2,2}$,

total of 27 portfolios. At this point, we simulated the preference of a DM in order to verify if among the portfolios we could assume one of them as completely satisfactory. Since this is not the case, we considered the portfolios that, according to our own interpretation of the University preferences, are relatively good. Consequently, in the Tables 11, 12 and 13 we also indicated the "good portfolios".

Applying the DRSA method to this preference information, we have obtained a total of 85 rules that were verified for at least the $50 \%$ of the "good" portfolios. Among those, only 9 rules were supported by all the "good" portfolios and only the following 4 decision rules contained two or more conditions in their "if" part:

Rule 1.1: if $F_{3,2}(\mathbf{x}) \geq 8$, and $F_{9,3}(\mathbf{x}) \geq 2$, then portfolio $P$ is "good", 
Table 11: The set of non-dominated portfolios obtained optimizing $P_{1, i}$.

\begin{tabular}{|c|c|c|c|c|c|c|c|c|c|c|}
\hline Portfolio & $F_{1,1}$ & $F_{2,1}$ & $F_{3,1}$ & $F_{4,1}$ & $F_{5,1}$ & $F_{6,1}$ & $F_{7,1}$ & $F_{8,1}$ & $F_{9,1}$ & Evaluation \\
\hline$P 1$ & 9 & 9 & 12 & 5 & 4 & 10 & 4 & 12 & 5 & Good \\
\hline$P 2$ & 9 & 9 & 12 & 5 & 4 & 10 & 4 & 12 & 5 & Good \\
\hline$P 3$ & 8 & 8 & 11 & 3 & 3 & 9 & 5 & 11 & 5 & Good \\
\hline$P 4$ & 9 & 9 & 12 & 5 & 4 & 10 & 4 & 12 & 5 & Good \\
\hline$P 5$ & 6 & 6 & 9 & 4 & 4 & 8 & 3 & 8 & 5 & $*$ \\
\hline$P 6$ & 4 & 4 & 8 & 5 & 4 & 7 & 1 & 7 & 5 & $*$ \\
\hline$P 7$ & 9 & 9 & 12 & 5 & 4 & 10 & 4 & 12 & 5 & Good \\
\hline$P 8$ & 9 & 9 & 12 & 5 & 4 & 10 & 4 & 12 & 5 & Good \\
\hline$P 9$ & 8 & 8 & 11 & 4 & 3 & 9 & 5 & 11 & 5 & Good \\
\hline$P 10$ & 7 & 7 & 9 & 5 & 5 & 7 & 3 & 9 & 6 & $*$ \\
\hline$P 11$ & 8 & 8 & 11 & 5 & 4 & 8 & 4 & 11 & 5 & Good \\
\hline$P 12$ & 9 & 9 & 12 & 5 & 4 & 10 & 4 & 12 & 5 & Good \\
\hline$P 13$ & 7 & 7 & 9 & 4 & 5 & 7 & 3 & 9 & 6 & $*$ \\
\hline$P 14$ & 9 & 9 & 12 & 5 & 4 & 10 & 4 & 12 & 5 & Good \\
\hline$P 15$ & 9 & 9 & 12 & 5 & 4 & 10 & 4 & 12 & 5 & Good \\
\hline$P 16$ & 9 & 9 & 12 & 5 & 4 & 10 & 4 & 12 & 5 & Good \\
\hline$P 17$ & 9 & 9 & 12 & 5 & 4 & 10 & 4 & 12 & 5 & Good \\
\hline$P 18$ & 9 & 9 & 12 & 5 & 4 & 10 & 4 & 12 & 5 & Good \\
\hline$P 19$ & 8 & 8 & 11 & 3 & 3 & 9 & 5 & 11 & 5 & Good \\
\hline$P 20$ & 8 & 8 & 11 & 3 & 3 & 9 & 5 & 11 & 5 & Good \\
\hline$P 21$ & 9 & 9 & 12 & 5 & 4 & 10 & 4 & 12 & 5 & Good \\
\hline$P 22$ & 9 & 9 & 12 & 5 & 4 & 10 & 4 & 12 & 5 & Good \\
\hline$P 23$ & 7 & 7 & 10 & 4 & 4 & 9 & 5 & 10 & 6 & $*$ \\
\hline$P 24$ & 8 & 8 & 11 & 3 & 3 & 9 & 5 & 11 & 5 & Good \\
\hline$P 25$ & 8 & 8 & 11 & 5 & 5 & 8 & 4 & 11 & 6 & Good \\
\hline$P 26$ & 8 & 8 & 11 & 3 & 35 & 9 & 5 & 11 & 5 & Good \\
\hline$P 27$ & 9 & 9 & 12 & 5 & 4 & 10 & 4 & 12 & 5 & Good \\
\hline
\end{tabular}


Table 12: The set of non-dominated portfolios presented obtained optimizing $P_{2, i}$.

\begin{tabular}{|c|c|c|c|c|c|c|c|c|c|c|}
\hline Portfolio & $F_{1,2}$ & $F_{2,2}$ & $F_{3,2}$ & $F_{4,2}$ & $F_{5,2}$ & $F_{6,2}$ & $F_{7,2}$ & $F_{8,2}$ & $F_{9,2}$ & Evaluation \\
\hline$P 1$ & 8 & 6 & 9 & 2 & 2 & 5 & 2 & 8 & 3 & Good \\
\hline$P 2$ & 8 & 6 & 9 & 2 & 2 & 5 & 2 & 8 & 3 & Good \\
\hline$P 3$ & 7 & 5 & 8 & 1 & 2 & 5 & 2 & 8 & 4 & Good \\
\hline$P 4$ & 8 & 6 & 9 & 2 & 2 & 5 & 2 & 8 & 3 & Good \\
\hline$P 5$ & 6 & 6 & 8 & 3 & 2 & 4 & 2 & 5 & 2 & * \\
\hline$P 6$ & 4 & 4 & 6 & 3 & 2 & 5 & 0 & 4 & 2 & $*$ \\
\hline$P 7$ & 8 & 6 & 9 & 2 & 2 & 5 & 2 & 8 & 3 & Good \\
\hline$P 8$ & 8 & 6 & 9 & 2 & 2 & 5 & 2 & 8 & 3 & Good \\
\hline$P 9$ & 7 & 5 & 8 & 2 & 2 & 4 & 2 & 8 & 4 & Good \\
\hline$P 10$ & 6 & 5 & 7 & 3 & 2 & 3 & 1 & 6 & 3 & $*$ \\
\hline$P 11$ & 7 & 5 & 8 & 3 & 2 & 4 & 2 & 7 & 3 & Good \\
\hline$P 12$ & 8 & 6 & 9 & 2 & 2 & 5 & 2 & 8 & 3 & Good \\
\hline$P 13$ & 6 & 5 & 7 & 2 & 2 & 4 & 1 & 6 & 3 & $*$ \\
\hline$P 14$ & 8 & 6 & 9 & 2 & 2 & 5 & 2 & 8 & 3 & Good \\
\hline$P 15$ & 8 & 6 & 9 & 2 & 2 & 5 & 2 & 8 & 3 & Good \\
\hline$P 16$ & 8 & 6 & 9 & 2 & 2 & 5 & 2 & 8 & 3 & Good \\
\hline$P 17$ & 8 & 6 & 9 & 2 & 2 & 5 & 2 & 8 & 3 & Good \\
\hline$P 18$ & 8 & 6 & 9 & 2 & 2 & 5 & 2 & 8 & 3 & Good \\
\hline$P 19$ & 7 & 5 & 8 & 1 & 2 & 5 & 2 & 8 & 4 & Good \\
\hline$P 20$ & 7 & 5 & 8 & 1 & 2 & 5 & 2 & 8 & 4 & Good \\
\hline$P 21$ & 8 & 6 & 9 & 2 & 2 & 5 & 2 & 8 & 3 & Good \\
\hline$P 22$ & 8 & 6 & 9 & 2 & 2 & 5 & 2 & 8 & 3 & Good \\
\hline$P 23$ & 6 & 5 & 7 & 1 & 2 & 4 & 2 & 9 & 4 & $*$ \\
\hline$P 24$ & 7 & 5 & 8 & 1 & 2 & 5 & 2 & 8 & 4 & Good \\
\hline$P 25$ & 7 & 5 & 8 & 1 & 2 & 5 & 2 & 8 & 4 & Good \\
\hline$P 26$ & 7 & 5 & 8 & 1 & $2^{36}$ & 5 & 2 & 8 & 4 & Good \\
\hline$P 27$ & 8 & 6 & 9 & 2 & 2 & 5 & 2 & 8 & 3 & Good \\
\hline
\end{tabular}


Table 13: The set of non-dominated portfolios obtained optimizing $P_{3, i}$.

\begin{tabular}{|c|c|c|c|c|c|c|c|c|c|c|}
\hline Portfolio & $F_{1,3}$ & $F_{2,3}$ & $F_{3,3}$ & $F_{4,3}$ & $F_{5,3}$ & $F_{6,3}$ & $F_{7,3}$ & $F_{8,3}$ & $F_{9,3}$ & Evaluation \\
\hline$P 1$ & 3 & 4 & 5 & 0 & 1 & 2 & 0 & 4 & 2 & Good \\
\hline$P 2$ & 3 & 4 & 5 & 0 & 1 & 2 & 0 & 4 & 2 & Good \\
\hline$P 3$ & 3 & 3 & 5 & 0 & 1 & 2 & 0 & 5 & 2 & Good \\
\hline$P 4$ & 3 & 4 & 5 & 0 & 1 & 2 & 0 & 4 & 2 & Good \\
\hline$P 5$ & 3 & 4 & 5 & 0 & 1 & 2 & 0 & 4 & 1 & $*$ \\
\hline$P 6$ & 1 & 4 & 3 & 0 & 1 & 2 & 0 & 2 & 1 & $*$ \\
\hline$P 7$ & 3 & 4 & 5 & 0 & 1 & 2 & 0 & 4 & 2 & Good \\
\hline$P 8$ & 3 & 4 & 5 & 0 & 1 & 2 & 0 & 4 & 2 & Good \\
\hline$P 9$ & 2 & 3 & 5 & 0 & 1 & 1 & 0 & 5 & 2 & Good \\
\hline$P 10$ & 1 & 4 & 3 & 0 & 1 & 1 & 0 & 3 & 2 & $*$ \\
\hline$P 11$ & 2 & 3 & 4 & 0 & 1 & 1 & 0 & 4 & 2 & Good \\
\hline$P 12$ & 3 & 4 & 5 & 0 & 1 & 2 & 0 & 4 & 2 & Good \\
\hline$P 13$ & 2 & 4 & 3 & 0 & 1 & 2 & 0 & 3 & 2 & $*$ \\
\hline$P 14$ & 3 & 4 & 5 & 0 & 1 & 2 & 0 & 4 & 2 & Good \\
\hline$P 15$ & 3 & 4 & 5 & 0 & 1 & 2 & 0 & 4 & 2 & Good \\
\hline$P 16$ & 3 & 4 & 5 & 0 & 1 & 2 & 0 & 4 & 2 & Good \\
\hline$P 17$ & 3 & 4 & 5 & 0 & 1 & 2 & 0 & 4 & 2 & Good \\
\hline$P 18$ & 3 & 4 & 5 & 0 & 1 & 2 & 0 & 4 & 2 & Good \\
\hline$P 19$ & 3 & 3 & 5 & 0 & 1 & 2 & 0 & 5 & 2 & Good \\
\hline$P 20$ & 3 & 3 & 5 & 0 & 1 & 2 & 0 & 5 & 2 & Good \\
\hline$P 21$ & 3 & 4 & 5 & 0 & 1 & 2 & 0 & 4 & 2 & Good \\
\hline$P 22$ & 3 & 4 & 5 & 0 & 1 & 2 & 0 & 4 & 2 & Good \\
\hline$P 23$ & 2 & 3 & 4 & 0 & 1 & 1 & 0 & 5 & 2 & $*$ \\
\hline$P 24$ & 3 & 3 & 5 & 0 & 1 & 2 & 0 & 5 & 2 & Good \\
\hline$P 25$ & 3 & 4 & 5 & 0 & 1 & 2 & 0 & 4 & 2 & Good \\
\hline$P 26$ & 3 & 3 & 5 & 0 & 37 & 2 & 0 & 5 & 2 & Good \\
\hline$P 27$ & 3 & 4 & 5 & 0 & 1 & 2 & 0 & 4 & 2 & Good \\
\hline
\end{tabular}


(if there are at least 8 projects that have evaluation "Medium" or better for criterion $g_{3}$, and at least 2 projects with evaluation " 500 " or better for criterion $g_{9}$, then the portfolio is good);

Rule 1.2: if $F_{7,1}(\mathbf{x}) \geq 4$, and $F_{3,2}(\mathbf{x}) \geq 8$, then portfolio $P$ is "good", (if there are at least 4 projects with evaluation "Medium" or better for criterion $g_{7}$, and at least 8 projects with evaluation "Medium" or better for criterion $g_{3}$, then the portfolio is good);

Rule 1.3: if $F_{3,2}(\mathbf{x}) \geq 8$ and $F_{8,2}(\mathbf{x}) \geq 7$, then strategy $\mathbf{x}$ is "good", (if there are at least 8 projects with evaluation "Medium" or better for criterion $g_{3}$, and at least 7 projects with evaluation "Medium" or better for criterion $g_{8}$, then the portfolio is good).

Rule 1.4: if $F_{3,2}(\mathbf{x}) \geq 8$ and $F_{9,2}(\mathbf{x}) \geq 3$, then strategy $\mathbf{x}$ is "good", (if there are at least 8 projects with evaluation "Medium" or better for criterion $g_{3}$, and at least 3 projects with evaluation "300" or better for criterion $g_{9}$, then the portfolio is good).

We selected Rule 1.2 as the most representative given that is concerning two criteria and two different quality thresholds, and we added the following constraints to the original optimization problem:

$$
\begin{aligned}
& \sum_{t \in T} F_{1,7, t}(\mathbf{x}) \geq 4, \\
& \sum_{t \in T} F_{2,3, t}(\mathbf{x}) \geq 8 .
\end{aligned}
$$




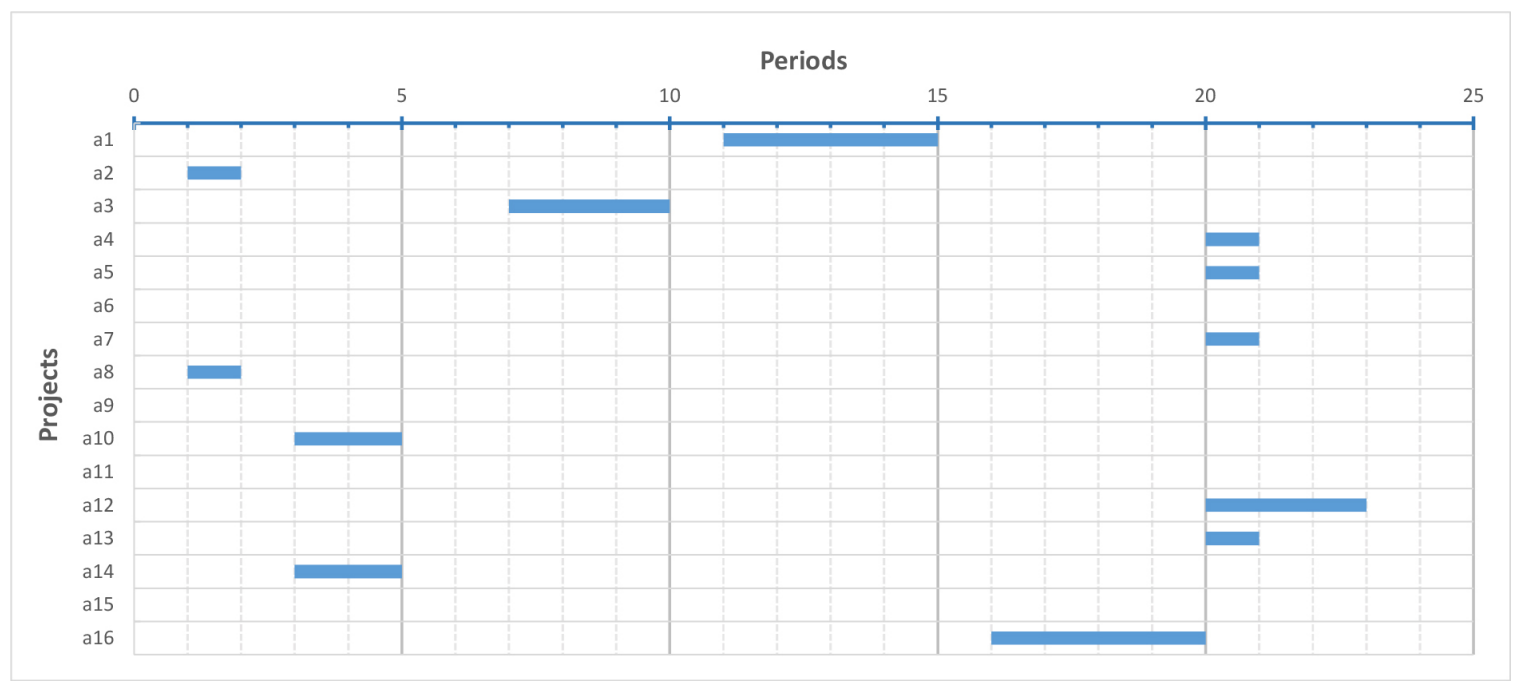

Figure 2: Selection and timing of the projects of the most preferred portftolio

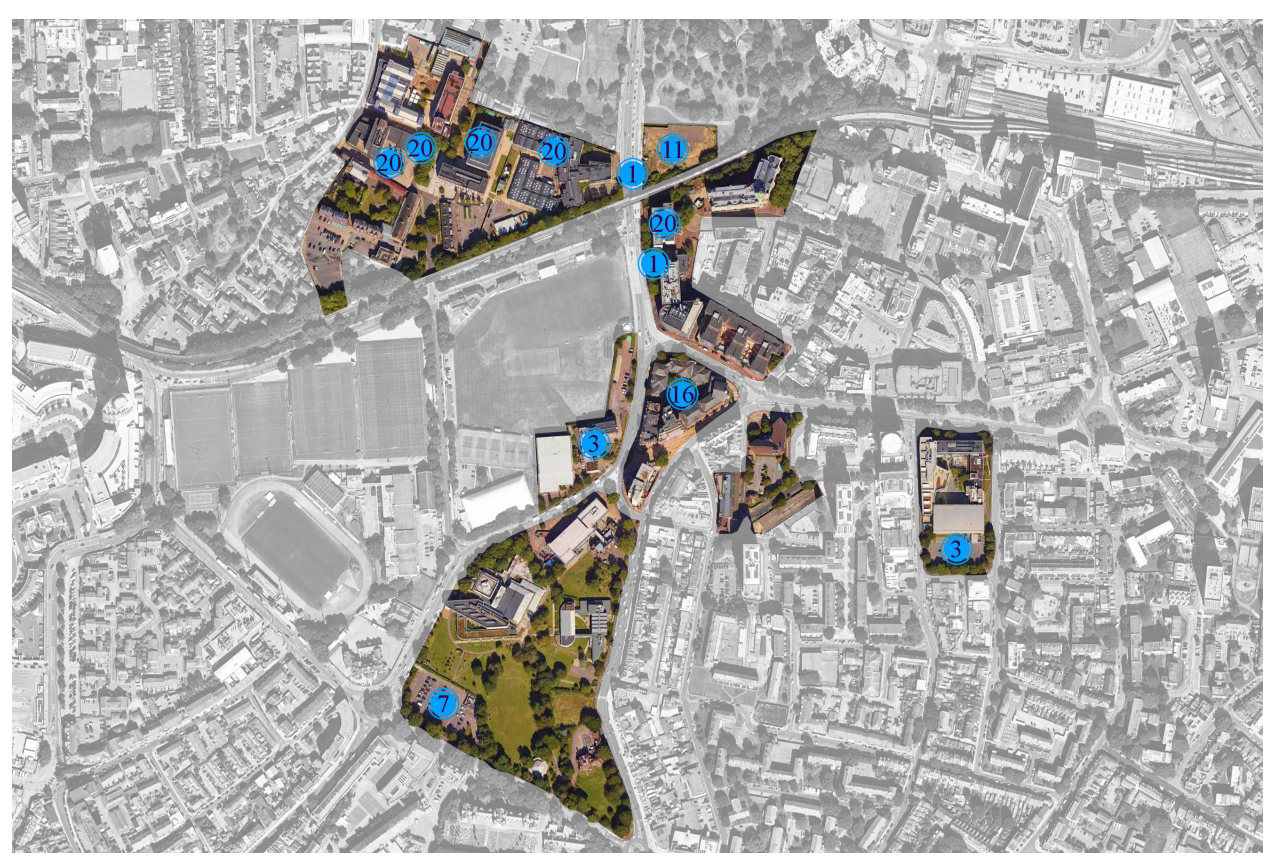

Figure 3: Position in the campus of the most preferred portfolio buildings and periods in which projects are scheduled to start 


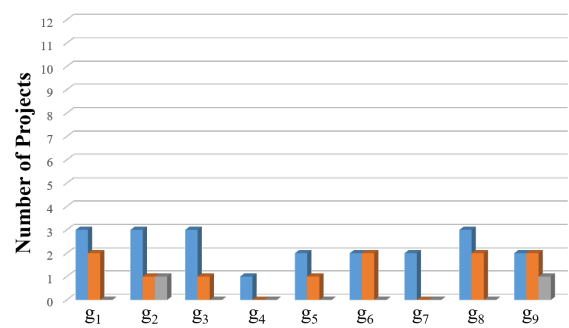

(a) Contribution in the first five years

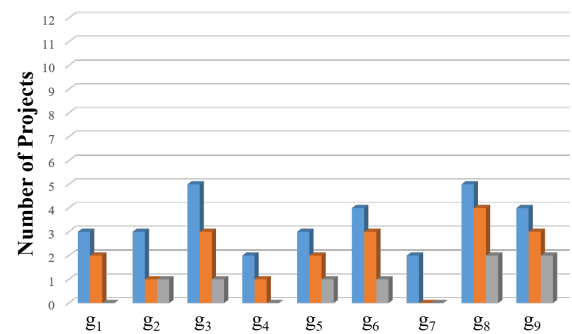

(c) Contribution in the first fifteen years

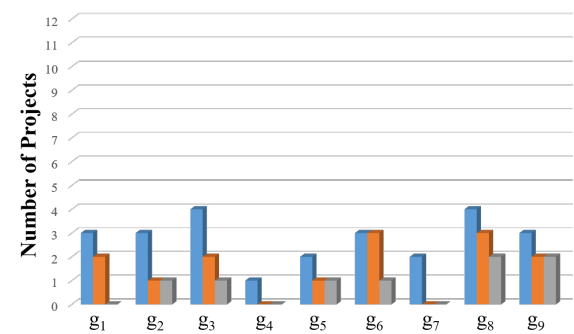

(b) Contribution in the first ten years

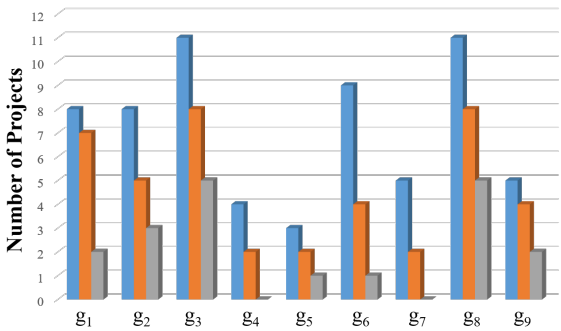

(d) Contribution in the first twenty years

First qualitative level $\square$ Second qualitative level $\square$ Third qualitative level

Figure 4: Histograms representing the set of non-dominated portfolios in the first iteration.

At this point a new set of portfolios is obtained applying the optimization model. For the sake of the space we do not report the portfolios obtained and the induced rules. Let us underline only that the obtained portfolios were very similar. In particular, portfolio $P 1$ was obtained when optimizing most of the considered objectives in the second iteration. Again, according to our understanding of the University preference. We gave an evaluation as "Good" an "Others" to the obtained porfolios and, applying DRSA, we induced decision rules explaining those judgments. Among the obtained rules we selected as the most representative the following one:

Rule 1.4: if $F_{6,1}(\mathbf{x}) \geq 9$, then strategy $\mathbf{x}$ is "good", 
(if there are at least 9 projects with evaluation at least "Low" for criterion $g_{6}$, then the portfolio is good).

We solved again the model with this additional constraint for every objective, and we obtained the same portfolio, in terms of projects included, although with two different timing. Therefore, the procedure stopped and one of these portfolios was considered the most preferred portfolio in this particular scenario. This portfolio, the most preferred one, is represented in Figures 2, 3 and 4. In particular, in Figure 2 we show the projects selected and their timing, i.e. when the scheduled projects will start and their duration, while in Figure 3 we highlight the position of the project in the campus map of the University of Portsmouth. Finally, in Figure 4 we report the cumulative $F_{i, c}$ every five years. For example, the $F_{1,1}$ in the histogram (a) in Figure 4 represents the number of projects that obtains an evaluation at least low on criterion $g_{1}$, that is, increasing the quality of the University Estate in terms of spaces, in the next first five years. Analogously, in the histogram $(d)$ in Figure $4 F_{1,1}$ represents the number of projects that obtains an evaluation at least low on criterion $g_{1}$ in the first twenty years of the plan.

\section{Conclusions}

In this paper we introduced a new model to deal with the selection and the scheduling of portfolios of urban planning projects. The model is capable to describe applications in which qualitative evaluations for a set of criteria can be handled, uncertainty can be modelled and the opinion of different stakeholders can be taken into account. The criteria can belong to a variety of categories that is essential nowadays when the focus is increasingly shifting 
from a merely economic point of view to the inclusion of more challenging criteria such as sustainability. We tested the model on a case study related to the development of the Master Plan of the University of Portsmouth showing how the model can support the decisions about which interventions and in which period they should be planned. The case study was selected for the emphasis that Universities are currently considering when planning their developments. One of the declared aims of the Master Plan is actually being a support for the sustainable growth of the city.

The model can be applied in an interactive way such that the stakeholders involved in the decision can construct the most preferred solution indicating and modifying their requirements throughout the interaction phase. Therefore, the adopted Master Plan taken can be better justified and explained to a diversified set of stakeholders.

We aim to further develop the model and to apply other multiobjective optimization techniques that can deal with the resolution of such models. Moreover, some other aspects could be considered such as the interaction among the criteria, related to the self strengthening and self weakening of evaluations on different criteria, or the interdependence of the projects, related to the self strengthening or the self weakening of the benefits of different projects. 


\section{References}

[1] P. Pujadas, F. Pardo-Bosch, A. Aguado-Renter, A. Aguado, MIVES multi-criteria approach for the evaluation, prioritization, and selection of public investment projects. A case study in the city of Barcelona, Land Use Policy 64 (2017) 29-37.

[2] A. Nesticò, M. R. Guarini, P. Morano, F. Sica, An economic analysis algorithm for urban forestry projects, Sustainability 11 (2019) 314.

[3] R. Z. Farahani, S. Fallah, R. Ruiz, S. Hosseini, N. Asgari, OR models in urban service facility location: a critical review of applications and future developments, European Journal of Operational Research 276 (2019) 1-27.

[4] H.-J. Wang, Z.-T. Zeng, A multi-objective decision-making process for reuse selection of historic buildings, Expert Systems with Applications 37 (2010) 1241-1249.

[5] I. Correia, T. Melo, A multi-period facility location problem with modular capacity adjustments and flexible demand fulfillment, Computers \& Industrial Engineering 110 (2017) 307-321.

[6] K. Deb, Multi-objective optimization, in: Search methodologies, Springer, 2014, pp. 403-449.

[7] A. Salo, J. Keisler, A. Morton, Portfolio decision analysis: improved methods for resource allocation, volume 162, Springer Science \& Business Media, 2011.

[8] M. Barbati, J. R. Figueira, S. Greco, A. Ishizaka, S. Panaro, A multiple 
criteria methodology for prioritizing and selecting portfolios of urban projects, arXiv preprint arXiv:1812.10410 (2018).

[9] N. J. Smelser, P. B. Baltes, et al., International encyclopedia of the social \& behavioral sciences, volume 11, Elsevier Amsterdam, 2001.

[10] D. Caparros-Midwood, S. Barr, R. Dawson, Optimised spatial planning to meet long term urban sustainability objectives, Computers, Environment and Urban Systems 54 (2015) 154-164.

[11] A. Nesticò, F. Sica, The sustainability of urban renewal projects: A model for economic multi-criteria analysis, Journal of Property Investment \& Finance 35 (2017) 397-409.

[12] R. Mosadeghi, J. Warnken, R. Tomlinson, H. Mirfenderesk, Comparison of fuzzy-AHP and AHP in a spatial multi-criteria decision making model for urban land-use planning, Computers, Environment and Urban Systems 49 (2015) 54-65.

[13] J. Li, Q. Pan, P. Duan, H. Sang, K. Gao, Solving multi-area environmental/economic dispatch by Pareto-based chemical-reaction optimization algorithm, IEEE/CAA Journal of Automatica Sinica (2017).

[14] A. Awasthi, S. S. Chauhan, S. K. Goyal, A multi-criteria decision making approach for location planning for urban distribution centers under uncertainty, Mathematical and Computer Modelling 53 (2011) 98-109.

[15] S. Kazazi Darani, A. Akbari Eslami, M. Jabbari, H. Asefi, Parking lot site selection using a fuzzy AHP-TOPSIS framework in tuyserkan, iran, 
Journal of Urban Planning and Development 144 (2018) 0401802204018022-10.

[16] R. Carli, M. Dotoli, R. Pellegrino, Multi-criteria decision-making for sustainable metropolitan cities assessment, Journal of Environmental Management 226 (2018) 46-61.

[17] K. Deb, A. Pratap, S. Agarwal, T. Meyarivan, A fast and elitist multiobjective genetic algorithm: NSGA-II, IEEE transactions on Evolutionary Computation 6 (2002) 182-197.

[18] M. Shaygan, A. Alimohammadi, A. Mansourian, Z. S. Govara, S. M. Kalami, Spatial multi-objective optimization approach for land use allocation using NSGA-II, IEEE Journal of Selected Topics in Applied Earth Observations and Remote Sensing 7 (2013) 906-916.

[19] Q. Kang, S. Feng, M. Zhou, A. C. Ammari, K. Sedraoui, Optimal load scheduling of plug-in hybrid electric vehicles via weight-aggregation multi-objective evolutionary algorithms, IEEE Transactions on Intelligent Transportation Systems 18 (2017) 2557-2568.

[20] X. Zuo, B. Li, X. Huang, M. Zhou, C. Cheng, X. Zhao, Z. Liu, Optimizing hospital emergency department layout via multiobjective tabu search, IEEE Transactions on Automation Science and Engineering 16 (2019) 1137-1147.

[21] V. Marinakis, H. Doukas, P. Xidonas, C. Zopounidis, Multicriteria decision support in local energy planning: an evaluation of alternative scenarios for the sustainable energy action plan, Omega 69 (2017) 1-16. 
[22] Y.-K. Juan, K. O. Roper, D. Castro-Lacouture, J. Ha Kim, Optimal decision making on urban renewal projects, Management Decision 48 (2010) 207-224.

[23] T. J. Lahtinen, R. P. Hämäläinen, J. Liesiö, Portfolio decision analysis methods in environmental decision making, Environmental modelling \& software 94 (2017) 73-86.

[24] A. Nesticò, P. Morano, F. Sica, A model to support the public administration decisions for the investments selection on historic buildings, Journal of Cultural Heritage 33 (2018) 201-207.

[25] C. Stummer, K. Heidenberger, Interactive R\&D portfolio analysis with project interdependencies and time profiles of multiple objectives, IEEE Transactions on Engineering Management 50 (2003) 175-183.

[26] M. Di Matteo, H. R. Maier, G. C. Dandy, Many-objective portfolio optimization approach for stormwater management project selection encouraging decision maker buy-in, Environmental modelling \& software 111 (2019) 340-355.

[27] F. Ghasemzadeh, N. Archer, P. Iyogun, A zero-one model for project portfolio selection and scheduling, Journal of the Operational Research Society 50 (1999) 745-755.

[28] F. Ghasemzadeh, N. P. Archer, Project portfolio selection through decision support, Decision Support Systems 29 (2000) 73-88.

[29] A. Zuluaga, J. A. Sefair, A. L. Medaglia, Model for the selection and scheduling of interdependent projects, in: 2007 IEEE Systems and Information Engineering Design Symposium, IEEE, pp. 1-7. 
[30] J. C. Pérez, M. H. Carrillo, J. R. Montoya-Torres, Multi-criteria approaches for urban passenger transport systems: a literature review, Annals of Operations Research 226 (2015) 69-87.

[31] S. Ghorbani, M. Rabbani, A new multi-objective algorithm for a project selection problem, Advances in Engineering Software 40 (2009) 9-14.

[32] A. L. Medaglia, D. Hueth, J. C. Mendieta, J. A. Sefair, A multiobjective model for the selection and timing of public enterprise projects, SocioEconomic Planning Sciences 42 (2008) 31-45.

[33] A. F. Carazo, T. Gómez, J. Molina, A. G. Hernández-Díaz, F. M. Guerrero, R. Caballero, Solving a comprehensive model for multiobjective project portfolio selection, Computers \& Operations research 37 (2010) 630-639.

[34] M. Barbati, S. Greco, M. Kadziński, R. Słowiński, Optimization of multiple satisfaction levels in portfolio decision analysis, Omega 78 (2018) 192-204.

[35] C. S. Park, G. Kim, S. Choi, Engineering economics, New Jersey: Pearson Prentice Hall (2007).

[36] J. M. Wong, S. T. Ng, Forecasting construction tender price index in hong kong using vector error correction model, Construction management and Economics 28 (2010) 1255-1268.

[37] M. Barbati, S. Corrente, S. Greco, A general space-time model for combinatorial optimization problems (and not only), Omega (2019). 
[38] R. Lahdelma, K. Miettinen, P. Salminen, Ordinal criteria in stochastic multicriteria acceptability analysis (SMAA), European Journal of Operational Research 147 (2003) 117-127.

[39] M. Ehrgott, X. Gandibleux, A survey and annotated bibliography of multiobjective combinatorial optimization, OR-Spektrum 22 (2000) 425-460.

[40] Q. Kang, X. Song, M. Zhou, L. Li, A collaborative resource allocation strategy for decomposition-based multiobjective evolutionary algorithms, IEEE Transactions on Systems, Man, and Cybernetics: Systems 49 (2018) 2416-2423.

[41] Z. Lv, L. Wang, Z. Han, J. Zhao, W. Wang, Surrogate-assisted particle swarm optimization algorithm with pareto active learning for expensive multi-objective optimization, IEEE/CAA Journal of Automatica Sinica 6 (2019) 838-849.

[42] J. Sun, S. Gao, H. Dai, J. Cheng, M. Zhou, J. Wang, Bi-objective elite differential evolution algorithm for multivalued logic networks, IEEE transactions on cybernetics 50 (2018) 233-246.

[43] Q. Peng, M. Zhou, Q. He, Y. Xia, C. Wu, S. Deng, Multi-objective optimization for location prediction of mobile devices in sensor-based applications, IEEE Access 6 (2018) 77123-77132.

[44] E. Karasakal, D. Nadirler, An interactive solution approach for a biobjective semi-desirable location problem, Journal of Global Optimization 42 (2008) 177-199. 
[45] K. Miettinen, F. Ruiz, A. P. Wierzbicki, Introduction to multiobjective optimization: interactive approaches, in: Multiobjective Optimization, Springer, 2008, pp. 27-57.

[46] M. Hartikainen, K. Miettinen, M. M. Wiecek, Constructing a pareto front approximation for decision making, Mathematical Methods of Operations Research 73 (2011) 209-234.

[47] C. G. da Silva, J. Meidanis, A. V. Moura, M. A. Souza, P. Viadanna Jr, M. R. de Oliveira, M. R. de Oliveira, L. H. Jardim, G. A. C. Lima, R. S. de Barros, An improved visualization-based approach for project portfolio selection, Computers in Human Behavior 73 (2017) 685-696.

[48] Y. Jiang, X. Liang, H. Liang, N. Yang, Multiple criteria decision making with interval stochastic variables: A method based on interval stochastic dominance, European Journal of Operational Research 271 (2018) 632-643.

[49] V. Gatta, E. Marcucci, P. Delle Site, M. Le Pira, C. S. Carrocci, Planning with stakeholders: Analysing alternative off-hour delivery solutions via an interactive multi-criteria approach, Research in Transportation Economics 73 (2019) 53-62.

[50] S. Greco, B. Matarazzo, R. Słowiński, Dominance-based rough set approach to interactive multiobjective optimization, in: Multiobjective optimization, Springer, 2008, pp. 121-155.

[51] S. Greco, B. Matarazzo, R. Slowinski, Rough sets theory for multicriteria decision analysis, European journal of operational research 129 (2001) 1-47. 
[52] S. Greco, B. Matarazzo, R. Słowiński, Dominance-based rough set approach to decision under uncertainty and time preference, Annals of Operations Research 176 (2010) 41-75.

[53] The Green Book. Central government guidance on appraisal and evaluation, HM Treasury, 2018.

[54] AECOM, Spon's architects' and builders' price book, CRC Press, Oakville, 143rd edit edition, 2018.

[55] S. Martello, D. Pisinger, P. Toth, New trends in exact algorithms for the 0-1 knapsack problem, European Journal of Operational Research 123 (2000) 325-332. 


\section{Appendix}

This Appendix is devoted to the presentation of some additional data used in the implementation of our model.

We list all the projects included in the Master Plan of the University of Portsmouth

1. New Anglesea Road crossing:

| Current state: Good; Intervention: Minor refurbishment |

Redevelopment of old crossing providing the resolution of conflicts between pedestrian and vehicles. This project provides an uninterrupted crossing for pedestrians.

2. New Anglesea Road crossing:

| Current state: Good; Intervention: Minor refurbishment |

Redevelopment of old crossing providing the resolution of conflicts between pedestrian and vehicles. This project provides an uninterrupted crossing for pedestrians.

3. New Sport building:

| Current state: New; Intervention: New building |

Building to gather a large number of activities improving the existing facilities. This brings a great satisfaction and generates increased income.

4. Richmond Lecture Theatre extension:

| Current state: New; Intervention: New building |

Building the extension of the Lecture Theatre providing new social, meeting and seminar spaces. Operating in conjunction with the exiting areas, it supplies a potential venue for conferences and events. 
5. Lion terrace and Richmond Square:

| Current state: Deficient; Intervention: Major refurbishment |

Refurbishing the areas creating a new courtyard increasing the identity and the quality of the environment. The redesign is an occasion to create new safe pathways and enhance the green mobility.

6. New Technology building:

| Current state: Good; Intervention: Demolition and rebuilt |

Demolishing two existing buildings to create a new tech-centre with a dynamic ground floor holding labs and workshop spaces. The new building provides the occasion to create an energetic frontage along Lion Terrace.

7. New Kings Henry's frontage and foyer:

| Current state: Good; Intervention: Minor refurbishment |

Refurbishment of the ground and first floor to create a generous, glazed foyer space with a clear view between the two side streets. The new frontage highlights the University presence within the city centre.

8. Cambridge road:

| Current state: Good; Intervention: Minor refurbishment |

Refurbishment of the sidewalk and of the pedestrian pathway creating more active public space and greater connections with the highway and the city.

9. New Student Hub and Union:

| Current state: Deficient; Intervention: Demolition and rebuilt |

Rebuilding or refurbishment of the Union creating new vibrant and interactive space for student to engage with seamless and providing a richer endowment of services in a single space. 
10. New Academic building:

| Current state: Good; Intervention: Demolition and rebuilt |

Supporting the redevelopment of the central area and increasing the available spaces for Sport Science linked to a refurbishment of the Spinnaker Sport Centre.

11. Science and Technology Quarter - Buildings 1-2:

| Current state: Good; Intervention: Demolition and rebuilt |

Demolishing two existing buildings to create two new structures releasing areas to new pedestrian routes to improve connectivity and identity of the areas. The atrium shapes allow to create more dynamic spaces.

12. Science and Technology Quarter - Buildings 3:

| Current state: Good; Intervention: Demolition and rebuilt |

Building a new structure crating a more coherent environment which faces with new public areas in a deep relation through the ground floor. Integration of functions creates flexible and stimulating environment.

13. Anglesea court:

| Current state: Deficient; Intervention: Major refurbishment |

Redeveloping this space creating a significant new public space integrated in the city. This creates a clear route across the campus with a mix of different outdoor spaces to relax, study and socialize.

14. Eldon bulging extension:

| Current state: New; Intervention: New building |

Building new extension of existent building reinforcing the faculty of Creative and Cultural Industries providing new social and pooled 
spaces for art exhibitions and performances.

15. New University house:

| Current state: Good; Intervention: Demolition and rebuilt |

Building the new University House reinforcing University presence in the city on one of its main highways.

16. St. Andrew's Court:

| Current state: Good; Intervention: Demolition and rebuilt |

Redevelopment to collect the opportunity to create a strong landmark in the city. The inclusion of pedestrian paths and a public courtyard, linked to an open ground floor, creates a greater environment.

We report in the following Table 14 how the budget is distributed for every year of the 5-years pattern.

Table 14: Budget scenarios

\begin{tabular}{|c|c|c|c|c|c|c|}
\hline Budget & & \multicolumn{5}{|c|}{5 -years pattern } \\
scenario & Tot & 1 & 2 & 3 & 4 & 5 \\
\hline \hline$b_{1}$ & 530 & 35 & 30 & 25 & 20 & 20 \\
\hline$b_{2}$ & 380 & 25 & 20 & 20 & 15 & 15 \\
\hline$b_{3}$ & 280 & 20 & 20 & 15 & 10 & 5 \\
\hline
\end{tabular}

